\title{
Existence and uniqueness of periodic solutions for some nonlinear fractional pantograph differential equations with $\psi$-Caputo derivative
}

Received: 29 May 2021 / Accepted: 7 September 2021 / Published online: 29 September 2021

(C) The Author(s) 2021

\begin{abstract}
The aim of this paper is to study the existence and uniqueness of periodic solutions for a certain type of nonlinear fractional pantograph differential equation with a $\psi$-Caputo derivative. The proofs are based on the coincidence degree theory of Mawhin. To show the efficiency of the results, some illustrative examples are included.
\end{abstract}

Mathematics Subject Classification $34 \mathrm{~A} 08 \cdot 34 \mathrm{~B} 10 \cdot 34 \mathrm{~B} 40$

\section{Introduction}

In last few decades, nonlinear fractional differential equations (NFDEs) have been the focus of many studies due to the intensive development of the theory of fractional calculus and to their frequent applications in many areas such as mechanics, physics, chemistry, engineering, and many other scientific disciplines $[15,16]$.

Recently, many definitions and results about fractional derivatives and integrals operators have been generalized $[1,2,4,17,22]$. Almeida [5,6] introduced a new generalized fractional derivative, the $\psi$-Caputo fractional derivative; some recent work on the subject of existence and uniqueness for NFDEs with $\psi$-Caputo fractional derivative can be found in $[7,13]$.

In the current paper, we study the nonlinear pantograph fractional equation with $\psi$-Caputo fractional derivative

$$
\begin{aligned}
{ }^{c} \mathfrak{D}_{0^{+}}^{\alpha ; \psi} u(t) & =h(t, u(t), u(\varepsilon t)), t \in J:=[0, \mathfrak{b}], \\
u(0) & =u(\mathfrak{b}),
\end{aligned}
$$

S. Bouriah · D. Foukrach

Department of Mathematics, Faculty of Exact Sciences and Informatics, University Hassiba Benbouali of Chlef, Chlef, Algeria

E-mail: s.bouriah@univ-chlef.dz

D. Foukrach

E-mail: d.foukrach@univ-chlef.dz

M. Benchohra

Laboratory of Mathematics, University of Sidi Bel-Abbes, P.O. Box 89, Sidi Bel-Abbés 22000, Algeria

E-mail: benchohra@yahoo.com

J. Graef $(\bowtie)$

Department of Mathematics, University of Tennessee at Chattanooga, Chattanooga, TN 37403, USA

E-mail: John-Graef@utc.edu 
where ${ }^{c} \mathfrak{D}_{0^{+}}^{\alpha ; \psi}$ denotes the $\psi$-Caputo fractional derivative of order $0<\alpha<1, \varepsilon \in(0,1)$, and $h: J \times \mathbb{R} \times \mathbb{R} \rightarrow \mathbb{R}$ is a continuous function.

Pantograph equations have been widely used in the fields of quantum mechanics and dynamical system $[20,21]$. Several researchers have investigated some new existence and uniqueness results for NFDE pantograph models and others by applying fixed point theorems, the nonlinear alternative on cones, or coincidence degree theory [3,8-12].

In [23], Shah et al. studied a class of $\psi$-Caputo fractional pantograph equations with nonlocal boundary conditions

$$
n u(0)+m u(\mathfrak{b})=\mathfrak{c},
$$

where $n, m$, and $\mathfrak{c}$ are real constants with $n+m \neq 0$, and obtained some existence and uniqueness results by using the Banach contraction theorem and Schaefer's fixed point theorem. However, if $n+m=0$, which includes the periodic case, the problem cannot be studied this way.

In this work, we construct a suitable operator and use the coincidence degree theory of Mawhin [14] to study the existence of solutions for NFDEs (1) with periodic boundary conditions (2).

The present article is organized as follows: In Sect. 2, some basic definitions and lemmas related to fractional calculus are recalled. In Sect. 3, the existence and uniqueness of periodic solutions for the NFDEs (1)-(2) are obtained. Finally, in Sect. 4, we give two examples to illustrate our main findings.

\section{Basic concepts}

We consider $C(J, \mathbb{R})$ and $C^{m}(J, \mathbb{R})$, the spaces of continuous and $m$ times continuously differentiable functions on $J$, respectively, with the supremum norm $\|\cdot\|_{\infty}$. We begin this section with the concept of a fractional integral or fractional derivative with respect to another function.

Definition 2.1 ([5]) Let $J=[0, \mathfrak{b}], 0<\mathfrak{b}<\infty$, be a finite or infinite interval, $\alpha>0, u$ be an integrable function defined on $J$, and $\psi \in C^{1}(J, \mathbb{R})$ be a positive increasing function, such that $\psi^{\prime}(t) \neq 0$ for all $t \in J$. Fractional integrals and fractional derivatives of a function $u$ with respect to another function $\psi$ are defined as

$$
\mathfrak{I}_{0^{+}}^{\alpha ; \psi} u(t):=\frac{1}{\Gamma(\alpha)} \int_{0}^{t} \psi^{\prime}(s)(\psi(t)-\psi(s))^{\alpha-1} u(s) \mathrm{d} s
$$

and

$$
\begin{aligned}
\mathfrak{D}_{0^{+}}^{\alpha ; \psi} u(t) & :=\left(\frac{1}{\psi^{\prime}(t)} \frac{1}{\mathrm{~d} t}\right)^{n} \Im_{0^{+}}^{n-\alpha ; \psi} u(t) \\
& =\frac{1}{\Gamma(n-\alpha)}\left(\frac{1}{\psi^{\prime}(t)} \frac{1}{\mathrm{~d} t}\right)^{n} \int_{0}^{t} \psi^{\prime}(s)(\psi(t)-\psi(s))^{n-\alpha-1} u(s) \mathrm{d} s,
\end{aligned}
$$

respectively, where $n=[\alpha]+1$.

In particular, if $0<\alpha<1$, we have

$$
\mathfrak{D}_{0^{+}}^{\alpha ; \psi} u(t)=\frac{1}{\Gamma(1-\alpha)}\left(\frac{1}{\psi^{\prime}(t)} \frac{1}{\mathrm{~d} t}\right)^{1} \int_{0}^{t} \psi^{\prime}(s)(\psi(t)-\psi(s))^{-\alpha} u(s) \mathrm{d} s .
$$

We will need several lemmas, some of which have appeared in the literature, to prove our main results.

Lemma 2.2 ([5]) Let $\alpha>0$ and $\beta>0$. Then, we have

$$
\mathfrak{I}_{0^{+}}^{\alpha ; \psi} \mathfrak{I}_{0^{+}}^{\beta ; \psi} u(t)=\mathfrak{I}_{0^{+}}^{\alpha+\beta ; \psi} u(t), \text { for all } t \in J .
$$

Lemma 2.3 ([17]) Let $\alpha>0, \rho>0$, and $t \in J$. If $u(t)=(\psi(t)-\psi(0))^{\rho-1}$, then

$$
\mathfrak{I}_{0^{+}}^{\alpha ; \psi} u(t)=\frac{\Gamma(\rho)}{\Gamma(\alpha+\rho)}(\psi(t)-\psi(0))^{\alpha+\rho-1} .
$$


Definition 2.4 ([5]) Let $n-1<\alpha<n$ with $n \in \mathbb{N}$ and $u, \psi \in C^{n}(J, \mathbb{R})$ be two functions, such that $\psi$ is increasing and positive with $\psi^{\prime}(t) \neq 0$ for any $t \in J$. The left $\psi$-Caputo fractional derivative of $u$ of order $\alpha$ is given by

$$
{ }^{c} \mathfrak{D}_{0^{+}}^{\alpha ; \psi} u(t):=\mathfrak{I}_{0^{+}}^{n-\alpha ; \psi}\left(\frac{1}{\psi^{\prime}(t)} \frac{\mathrm{d}}{\mathrm{d} t}\right)^{n} u(t), t \in J .
$$

In particular, if $0<\alpha<1$, we have

$$
{ }^{c} \mathfrak{D}_{0^{+}}^{\alpha ; \psi} u(t)=\frac{1}{\Gamma(1-\alpha)} \int_{0}^{t}(\psi(t)-\psi(s))^{-\alpha} \mathfrak{u}^{\prime}(s) \mathrm{d} s, t \in J .
$$

Lemma 2.5 ([5]) If $u \in C^{n}(J, \mathbb{R})$ and $n-1<\alpha<n$, then

$$
\mathfrak{I}_{0^{+}}^{\alpha ; \psi} \mathfrak{D}_{0^{+}}^{\alpha ; \psi} u(t)=u(t)-\sum_{k=0}^{n-1} \frac{(\psi(t)-\psi(0))^{k}}{k !}\left(\frac{1}{\psi^{\prime}(t)} \frac{d}{d t}\right)^{k} u(0) .
$$

In particular, when $0<\alpha<1$, we have

$$
\mathfrak{I}_{0^{+}}^{\alpha ; \psi} \mathfrak{D}_{0^{+}}^{\alpha ; \psi} u(t)=u(t)-u(0) .
$$

Lemma 2.6 ([5]) If $u \in C^{1}(J, \mathbb{R})$ and $\alpha>0$, we have

$$
{ }^{c} \mathfrak{D}_{0^{+}}^{\alpha ; \psi} \mathfrak{I}_{0^{+}}^{\alpha ; \psi} u(t)=u(t) .
$$

Lemma 2.7 ([5]) Let $u, v \in C^{1}(J, \mathbb{R})$ and $0<\alpha<1$. Then

$$
{ }^{c} \mathfrak{D}_{0^{+}}^{\alpha ; \psi} u(t)={ }^{c} \mathfrak{D}_{0^{+}}^{\alpha ; \psi} v(t) \text { if and only if } u(t)=v(t)+(u-v)(0) .
$$

Remark 2.8 If $v \equiv 0$, then

$$
{ }^{c} \mathfrak{D}_{0^{+}}^{\alpha, \beta ; \psi} u(t)=0 \text { if and only if } u(t)=u(0) .
$$

Next, we present definitions and concepts from coincidence degree theory that are essential in the proofs of our results (see $[14,18])$.

Definition 2.9 Let $\mathcal{X}$ and $\mathcal{Y}$ be normed spaces. A Fredholm operator of index zero is a linear operator $\mathfrak{L}$ : $\operatorname{Dom}(\mathfrak{L}) \subset \mathcal{X} \rightarrow \mathcal{Y}$, such that

(a) $\operatorname{dim} \operatorname{ker} \mathfrak{L}=\operatorname{codim} \operatorname{Img} \mathfrak{L}<+\infty$.

(b) Img $\mathfrak{L}$ is a closed subset of $\mathcal{Y}$.

By Definition 2.9, there exist continuous projectors $Q: \mathcal{Y} \rightarrow \mathcal{Y}$ and $\mathcal{P}: \mathcal{X} \rightarrow \mathcal{X}$ satisfying

$$
\operatorname{Img} \mathfrak{L}=\operatorname{ker} Q, \quad \operatorname{ker} \mathfrak{L}=\operatorname{Img} \mathcal{P}, \quad \mathcal{Y}=\operatorname{Img} Q \oplus \operatorname{Img} \mathfrak{L}, \quad \mathcal{X}=\operatorname{ker} \mathcal{P} \oplus \operatorname{ker} \mathfrak{L} .
$$

Thus, the restriction of $\mathfrak{L}$ to Dom $\mathfrak{L} \cap \operatorname{ker} \mathcal{P}$, denoted by $\mathfrak{L}_{\mathcal{P}}$, is an isomorphism onto its image.

Definition 2.10 Let $\Omega \subseteq \mathcal{X}$ be a bounded subset and $\mathfrak{L}$ be a Fredholm operator of index zero with Dom $\mathfrak{L} \cap \Omega$ $\emptyset$. Then, the operator $\mathcal{N}: \bar{\Omega} \rightarrow \mathcal{Y}$ is said to be $\mathfrak{L}$-compact in $\bar{\Omega}$ if

(a) the mapping $Q \mathcal{N}: \bar{\Omega} \rightarrow \mathcal{Y}$ is continuous and $Q \mathcal{N}(\bar{\Omega}) \subseteq \mathcal{Y}$ is bounded.

(b) the mapping $\left(\mathfrak{L}_{\mathcal{P}}\right)^{-1}(i d-Q) \mathcal{N}: \bar{\Omega} \rightarrow \mathcal{X}$ is completely continuous.

Lemma 2.11 ([19]) Let $\mathcal{X}$ and $\mathcal{Y}$ be Banach spaces and $\Omega \subset \mathcal{X}$ be a bounded open set that is symmetric with $0 \in \Omega$. Suppose that $\mathfrak{L}:$ Dom $\mathfrak{L} \subset \mathcal{X} \rightarrow \mathcal{Y}$ is a Fredholm operator of index zero with Dom $\mathfrak{L} \cap \bar{\Omega} \neq \emptyset$, and $\mathcal{N}: \mathcal{X} \rightarrow \mathcal{Y}$ is a $\mathfrak{L}$-compact operator on $\bar{\Omega}$. Assume, moreover, that

$$
\mathfrak{L} x-\mathcal{N} x \neq-\zeta(\mathfrak{L} x+\mathcal{N}(-x))
$$

for any $x \in$ Dom $\mathfrak{L} \cap \partial \Omega$ and any $\zeta \in(0,1]$, where $\partial \Omega$ is the boundary of $\Omega$ with respect to $\mathcal{X}$. Then, there exists at least one solution of the equation $\mathfrak{L} x=\mathcal{N} x$ on Dom $\mathfrak{L} \cap \bar{\Omega}$. 


\section{Main results}

Let the spaces

$$
\mathcal{X}=\left\{u \in C(J, \mathbb{R}): u(t)=\mathfrak{I}_{0^{+}}^{\alpha ; \psi} v(t), v \in C(J, \mathbb{R})\right\}
$$

and

$$
\mathcal{Y}=C(J, \mathbb{R}),
$$

be endowed with the norms

$$
\|u\|_{\mathcal{X}}=\|u\|_{\mathcal{Y}}=\|u\|_{\infty}=\sup _{t \in J}|u(t)| .
$$

We define the operator $\mathfrak{L}:$ Dom $\mathfrak{L} \subseteq \mathcal{X} \rightarrow \mathcal{Y}$ by

$$
\mathfrak{L} u:={ }^{c} \mathfrak{D}_{0^{+}}^{\alpha ; \psi} u,
$$

where

$$
\operatorname{Dom} \mathfrak{L}=\left\{u \in \mathcal{X}:{ }^{c} \mathfrak{D}_{0^{+}}^{\alpha ; \psi} u \in \mathcal{Y}, u(0)=u(\mathfrak{b})\right\} .
$$

Lemma 3.1 For the operator $\mathfrak{L}$ given in (3), we have

$$
\text { ker } \mathfrak{L}=\{u \in \mathcal{X}: u(t)=u(0), t \in J\}
$$

and

$$
\operatorname{Img} \mathfrak{L}=\left\{v \in \mathcal{Y}: \int_{0}^{\mathfrak{b}} \psi^{\prime}(s)(\psi(\mathfrak{b})-\psi(s))^{\alpha-1} v(s) d s=0\right\} .
$$

Proof By Remark 2.8, we have for all $u \in \mathcal{X}$ the equation $\mathfrak{L} u={ }^{c} \mathfrak{D}_{0^{+}}^{\alpha ; \psi} u=0$ in $J$, has a solution of the form

$$
u(t)=c_{0}=u(0), t \in J
$$

so

$$
\text { ker } \mathfrak{L}=\{u \in \mathcal{X}: u(t)=u(0), t \in J\} .
$$

For $v \in \operatorname{Img} \mathfrak{L}$, there exists $u \in \operatorname{Dom} \mathfrak{L}$, such that $v=\mathfrak{L} u \in \mathcal{Y}$. From Lemma 2.5, we obtain that for every $t \in J$

$$
u(t)=u(0)+\Im_{0^{+}}^{\alpha ; \psi} v(t)=u(0)+\frac{1}{\Gamma(\alpha)} \int_{0}^{t} \psi^{\prime}(s)(\psi(t)-\psi(s))^{\alpha-1} v(s) \mathrm{d} s .
$$

Since $u \in \operatorname{Dom} \mathfrak{L}$, we have $u(0)=u(\mathfrak{b})$. Thus

$$
\int_{0}^{\mathfrak{b}} \psi^{\prime}(s)(\psi(\mathfrak{b})-\psi(s))^{\alpha-1} v(s) \mathrm{d} s=0 .
$$

Furthermore, if $v \in \mathcal{Y}$ satisfies

$$
\int_{0}^{\mathfrak{b}} \psi^{\prime}(s)(\psi(\mathfrak{b})-\psi(s))^{\alpha-1} v(s) \mathrm{d} s=0,
$$

then for any $u(t)=\mathfrak{I}_{0^{+}}^{\alpha ; \psi} v(t)$, using Lemma 2.6, we obtain $v(t)={ }^{c} \mathfrak{D}_{0^{+}}^{\alpha ; \psi} u(t)$. Therefore

$$
u(\mathfrak{b})=u(0),
$$

which implies that $u \in$ Dom $\mathfrak{L}$, and so, $v \in \operatorname{Img} \mathfrak{L}$. Hence

$$
\operatorname{Img} \mathfrak{L}=\left\{v \in \mathcal{Y}: \int_{0}^{\mathfrak{b}} \psi^{\prime}(s)(\psi(\mathfrak{b})-\psi(s))^{\alpha-1} v(s) \mathrm{d} s=0\right\},
$$

which completes the proof of the lemma. 
Lemma 3.2 Let $\mathfrak{L}$ be defined by (3). Then, $\mathfrak{L}$ is a Fredholm operator of index zero, and the linear continuous projector operators $Q: \mathcal{Y} \rightarrow \mathcal{Y}$ and $\mathcal{P}: \mathcal{X} \rightarrow \mathcal{X}$ can be written as

$$
Q(v)=\frac{\alpha}{(\psi(\mathfrak{b})-\psi(0))^{\alpha}} \int_{0}^{\mathfrak{b}} \psi^{\prime}(s)(\psi(\mathfrak{b})-\psi(s))^{\alpha-1} v(s) d s
$$

and

$$
\mathcal{P}(u)=u(0) .
$$

Furthermore, the operator $\mathfrak{L}_{\mathcal{P}}^{-1}: \operatorname{Img} \mathfrak{L} \rightarrow \mathcal{X} \cap \operatorname{ker} \mathcal{P}$ becomes

$$
\mathfrak{L}_{\mathcal{P}}^{-1}(v)(t)=\mathfrak{I}_{0^{+}}^{\alpha ; \psi} v(t), t \in J .
$$

Proof Clearly, for each $v \in \mathcal{Y}, Q^{2} v=Q v$ and $v=Q(v)+(v-Q(v))$, where $(v-Q(v)) \in \operatorname{ker} Q=\operatorname{Img} \mathfrak{L}$. Using these facts, we see that $\operatorname{Img} Q \cap \operatorname{Img} \mathfrak{L}=0$, so

$$
\mathcal{Y}=\operatorname{Img} \mathfrak{L} \oplus \operatorname{Img} Q .
$$

Similarly, $\operatorname{Img} \mathcal{P}=\operatorname{ker} \mathfrak{L}$ and $\mathcal{P}^{2}=\mathcal{P}$. It follows that for each $u \in \mathcal{X}, u=(u-\mathcal{P}(u))+\mathcal{P}(u)$, so $\mathcal{X}=\operatorname{ker} \mathcal{P}+\operatorname{ker} \mathfrak{L}$. Clearly, we have $\operatorname{ker} \mathcal{P} \cap \operatorname{ker} \mathfrak{L}=0$. Hence

$$
\mathcal{X}=\operatorname{ker} \mathcal{P} \oplus \operatorname{ker} \mathfrak{L} .
$$

Therefore

$$
\operatorname{dim} \operatorname{ker} \mathfrak{L}=\operatorname{dim} \operatorname{Img} Q=\operatorname{codim} \operatorname{Img} \mathfrak{L} .
$$

Consequently, $\mathfrak{L}$ is a Fredholm operator of index zero.

Now, we will show that the inverse of $\left.\mathfrak{L}\right|_{\text {Dom } \mathfrak{L} \cap \text { ker } \mathcal{P}}$ is $\mathfrak{L}_{\mathcal{P}}^{-1}$. For $v \in \operatorname{Img} \mathfrak{L}$, by Lemma 2.6, we have

$$
\mathfrak{L}_{\mathcal{P}}^{-1}(v)={ }^{c} \mathfrak{D}_{0^{+}}^{\alpha ; \psi}\left(\mathfrak{I}_{0^{+}}^{\alpha ; \psi} v\right)=v .
$$

In addition, for $u \in \operatorname{Dom} \mathfrak{L} \cap \operatorname{ker} \mathcal{P}$,

$$
\mathfrak{L}_{\mathcal{P}}^{-1}(\mathfrak{L}(u(t)))=\mathfrak{I}_{0^{+}}^{\alpha ; \psi}\left({ }^{c} \mathfrak{D}_{0^{+}}^{\alpha ; \psi} u(t)\right)=u(t)-u(0), t \in J .
$$

Using the fact that $u \in \operatorname{Dom} \mathfrak{L} \cap \operatorname{ker} \mathcal{P}$, we see that

$$
u(0)=0 .
$$

Thus

$$
\mathfrak{L}_{\mathcal{P}}^{-1} \mathfrak{L}(u)=u .
$$

From (4) and (5), it follows that $\mathfrak{L}_{\mathcal{P}}^{-1}=\left(\left.\mathfrak{L}\right|_{\text {Dom } \mathfrak{L} \cap \operatorname{ker} \mathcal{P}}\right)^{-1}$, which completes the proof.

We will need the following condition in the sequel.

(H1) There exist positive constants $\gamma$ and $\eta$, such that

$$
|h(t, u, v)-h(t, \bar{u}, \bar{v})| \leqslant \gamma|u-\bar{u}|+\eta|v-\bar{v}|,
$$

for every $t \in J$ and $u, \bar{u}, v, \bar{v} \in \mathbb{R}$.

Define $\mathcal{N}_{\varepsilon}: \mathcal{X} \rightarrow \mathcal{Y}$ by

$$
\mathcal{N}_{\varepsilon} u(t):=h(t, u(t), u(\varepsilon t)), t \in J \text { and } \varepsilon \in(0,1) .
$$

Then, the problem (1)-(2) is equivalent to the problem $\mathfrak{L} u(t)=\mathcal{N}_{\varepsilon} u(t), t \in J$ and $u \in \mathcal{X}$.

Lemma 3.3 If (H1) holds, then for any bounded open set $\Omega \subset \mathcal{X}$, the operator $\mathcal{N}_{\varepsilon}$ is $\mathfrak{L}$-compact. 
Proof For $\mathcal{M}>0$, consider the bounded open set $\Omega=\left\{u \in \mathcal{X}:\|u\|_{\mathcal{X}}<\mathcal{M}\right\}$. We divide the proof into three steps.

Step 1: $\mathcal{Q} \mathcal{N}_{\varepsilon}$ is continuous. Let $\left(u_{n}\right)_{\mathfrak{n} \in \mathbb{N}}$ be a sequence, such that $u_{n} \rightarrow u$ in $\mathcal{Y}$; then, for each $t \in J$, we have

$$
\begin{aligned}
\mid \mathcal{Q N}_{\varepsilon}\left(u_{n}\right)(t) & -\mathcal{Q N}_{\varepsilon}(u)(t) \mid \\
& \leqslant \frac{\alpha}{(\psi(\mathfrak{b})-\psi(0))^{\alpha}} \int_{0}^{\mathfrak{b}} \psi^{\prime}(s)(\psi(\mathfrak{b})-\psi(s))^{\alpha-1}\left|\mathcal{N}_{\varepsilon}\left(u_{n}\right)(s)-\mathcal{N}_{\varepsilon}(u)(s)\right| \mathrm{d} s .
\end{aligned}
$$

By $(H 1)$, we have

$$
\begin{aligned}
\mid \mathcal{Q} \mathcal{N}_{\varepsilon}\left(u_{n}\right)(t)- & \mathcal{Q} \mathcal{N}_{\varepsilon}(u)(t) \mid \\
\leqslant & \frac{\alpha \gamma}{(\psi(\mathfrak{b})-\psi(0))^{\alpha}} \int_{0}^{\mathfrak{b}} \psi^{\prime}(s)(\psi(\mathfrak{b})-\psi(s))^{\alpha-1}\left|u_{n}(s)-u(s)\right| \mathrm{d} s \\
& +\frac{\alpha \eta}{(\psi(\mathfrak{b})-\psi(0))^{\alpha}} \int_{0}^{\mathfrak{b}} \psi^{\prime}(s)(\psi(\mathfrak{b})-\psi(s))^{\alpha-1}\left|u_{n}(\varepsilon s)-u(\varepsilon s)\right| \mathrm{d} s \\
\leqslant & \frac{\alpha(\gamma+\eta)\left\|u_{n}-\mathfrak{u}\right\| \mathcal{Y}}{(\psi(\mathfrak{b})-\psi(0))^{\alpha}} \int_{0}^{\mathfrak{b}} \psi^{\prime}(s)(\psi(\mathfrak{b})-\psi(s))^{\alpha-1} \mathrm{~d} s \\
\leqslant & (\gamma+\eta)\left\|u_{n}-\mathfrak{u}\right\| \mathcal{Y} .
\end{aligned}
$$

Thus, for each $t \in J$

$$
\left|\mathcal{Q} \mathcal{N}_{\varepsilon}\left(u_{n}\right)(t)-\mathcal{Q} \mathcal{N}_{\varepsilon}(u)(t)\right| \rightarrow 0 \text { as } n \rightarrow+\infty,
$$

and hence

$$
\left\|\mathcal{Q} \mathcal{N}_{\varepsilon}\left(u_{n}\right)-\mathcal{Q N}_{\varepsilon}(u)\right\| \mathcal{Y} \rightarrow 0 \text { as } n \rightarrow+\infty .
$$

Therefore, $\mathcal{Q \mathcal { N } _ { \varepsilon }}$ is continuous.

Step 2: $\mathcal{Q} \mathcal{N}_{\varepsilon}(\bar{\Omega})$ is bounded. For $t \in J$ and $u \in \bar{\Omega}$, we have

$$
\begin{aligned}
\left|\mathcal{Q N}_{\varepsilon}(u)(t)\right| \leqslant & \frac{\alpha}{(\psi(\mathfrak{b})-\psi(0))^{\alpha}} \int_{0}^{\mathfrak{b}} \psi^{\prime}(s)(\psi(\mathfrak{b})-\psi(s))^{\alpha-1}\left|\mathcal{N}_{\varepsilon}(u)(s)\right| \mathrm{d} s \\
\leqslant & \frac{\alpha}{(\psi(\mathfrak{b})-\psi(0))^{\alpha}} \\
& \times \int_{0}^{\mathfrak{b}} \psi^{\prime}(s)(\psi(\mathfrak{b})-\psi(s))^{\alpha-1}|h(s, u(s), u(\varepsilon s))-h(s, 0,0)| \mathrm{d} s \\
& +\frac{\alpha}{(\psi(\mathfrak{b})-\psi(0))^{\alpha}} \int_{0}^{\mathfrak{b}} \psi^{\prime}(s)(\psi(\mathfrak{b})-\psi(s))^{\alpha-1}|h(s, 0,0)| \mathrm{d} s \\
\leqslant & h^{*}+\frac{\alpha \gamma}{(\psi(\mathfrak{b})-\psi(0))^{\alpha}} \int_{0}^{\mathfrak{b}} \psi^{\prime}(s)(\psi(\mathfrak{b})-\psi(s))^{\alpha-1}|u(s)| \mathrm{d} s \\
& +\frac{\alpha \eta}{(\psi(\mathfrak{b})-\psi(0))^{\alpha}} \int_{0}^{\mathfrak{b}} \psi^{\prime}(s)(\psi(\mathfrak{b})-\psi(s))^{\alpha-1}|u(\varepsilon s)| \mathrm{d} s \\
\leqslant & h^{*}+(\gamma+\eta) \mathcal{M},
\end{aligned}
$$

where $h^{*}=\|h(\cdot, 0,0)\|_{\infty}$. Thus

$$
\left\|\mathcal{Q} \mathcal{N}_{\varepsilon}(u)\right\| \mathcal{Y} \leqslant h^{*}+(\gamma+\eta) \mathcal{M}
$$

so $\mathcal{Q N}(\bar{\Omega})$ is a bounded set in $\mathcal{Y}$.

Step 3: $\mathfrak{L}_{\mathcal{P}}^{-1}(i d-Q) \mathcal{N}_{\varepsilon}: \bar{\Omega} \rightarrow \mathcal{X}$ is completely continuous. We will use the Arzelà-Ascoli theorem, so we need to show that $\mathfrak{L}_{\mathcal{P}}^{-1}(i d-Q) \mathcal{N}_{\varepsilon}(\bar{\Omega}) \subset \mathcal{X}$ is uniformly bounded and equicontinuous. First, for any $u \in \bar{\Omega}$ and $t \in J$ 


$$
\begin{aligned}
\mathfrak{L}_{\mathcal{P}}^{-1} & \left(\mathcal{N}_{\varepsilon} u(t)-\mathcal{Q N} \mathcal{N}_{\varepsilon} u(t)\right) \\
= & \mathfrak{I}_{0^{+}}^{\alpha ; \psi}\left[h(t, u(t), u(\varepsilon t))-\frac{\alpha}{(\psi(\mathfrak{b})-\psi(0))^{\alpha}} \int_{0}^{\mathfrak{b}} \psi^{\prime}(s)(\psi(\mathfrak{b})-\psi(s))^{\alpha-1} h(s, u(s), u(\varepsilon s)) \mathrm{d} s\right] \\
= & \frac{1}{\Gamma(\alpha)} \int_{0}^{t} \psi^{\prime}(s)(\psi(t)-\psi(s))^{\alpha-1} h(s, u(s), u(\varepsilon s)) \mathrm{d} s \\
& -\frac{(\psi(t)-\psi(0))^{\alpha}}{\Gamma(\alpha)(\psi(\mathfrak{b})-\psi(0))^{\alpha}} \int_{0}^{\mathfrak{b}} \psi^{\prime}(s)(\psi(\mathfrak{b})-\psi(s))^{\alpha-1} h(s, u(s), u(\varepsilon s)) \mathrm{d} s \\
\leqslant & \frac{1}{\Gamma(\alpha)} \int_{0}^{t} \psi^{\prime}(s)(\psi(t)-\psi(s))^{\alpha-1}|h(s, u(s), u(\varepsilon s))-h(s, 0,0)| \mathrm{d} s \\
& +\frac{1}{\Gamma(\alpha)} \int_{0}^{t} \psi^{\prime}(s)(\psi(t)-\psi(s))^{\alpha-1}|h(s, 0,0)| \mathrm{d} s \\
& +\frac{1}{\Gamma(\alpha)} \int_{0}^{\mathfrak{b}} \psi^{\prime}(s)(\psi(\mathfrak{b})-\psi(s))^{\alpha-1}|h(s, u(s), u(\varepsilon s))-h(s, 0,0)| \mathrm{d} s \\
& +\frac{1}{\Gamma(\alpha)} \int_{0}^{\mathfrak{b}} \psi^{\prime}(s)(\psi(\mathfrak{b})-\psi(s))^{\alpha-1}|h(s, 0,0)| \mathrm{d} s, \\
\leqslant & \frac{2 h^{*}}{\alpha \Gamma(\alpha)}(\psi(\mathfrak{b})-\psi(0))^{\alpha}+\frac{\gamma}{\Gamma(\alpha)} \int_{0}^{t} \psi^{\prime}(s)(\psi(t)-\psi(s))^{\alpha-1}|u(s)| \mathrm{d} s \\
& +\frac{\eta}{\Gamma(\alpha)} \int_{0}^{t} \psi^{\prime}(s)(\psi(t)-\psi(s))^{\alpha-1}|u(\varepsilon s)| \mathrm{d} s \\
& +\frac{\gamma}{\Gamma(\alpha)} \int_{0}^{\mathfrak{b}} \psi^{\prime}(s)(\psi(\mathfrak{b})-\psi(s))^{\alpha-1}|u(s)| \mathrm{d} s \\
& +\frac{\eta}{\Gamma(\alpha)} \int_{0}^{\mathfrak{b}} \psi^{\prime}(s)(\psi(\mathfrak{b})-\psi(s))^{\alpha-1}|u(\varepsilon s)| \mathrm{d} s \\
\leqslant & \frac{2(\psi(\mathfrak{b})-\psi(0))^{\alpha}}{\Gamma(\alpha+1)}\left[h^{*}+(\gamma+\eta) \mathcal{M}\right] .
\end{aligned}
$$

Therefore

$$
\left\|\mathfrak{L}_{\mathcal{P}}^{-1}(i d-Q) \mathcal{N}_{\varepsilon} u\right\|_{\mathcal{X}} \leqslant \frac{2(\psi(\mathfrak{b})-\psi(0))^{\alpha}}{\Gamma(\alpha+1)}\left[h^{*}+(\gamma+\eta) \mathcal{M}\right],
$$

which means that $\mathfrak{L}_{\mathcal{P}}^{-1}(i d-Q) \mathcal{N}_{\varepsilon}(\bar{\Omega})$ is uniformly bounded on $\mathcal{X}$.

To show that $\mathfrak{L}_{\mathcal{P}}^{-1}(i d-Q) \mathcal{N}_{\varepsilon}(\bar{\Omega})$ is equicontinuous, let $0<t_{1}<t_{2} \leqslant \mathfrak{b}$ and $u \in \bar{\Omega}$. Then

$$
\begin{aligned}
\mid \mathfrak{L}_{\mathcal{P}}^{-1}(i d- & Q) \mathcal{N}_{\varepsilon} u\left(t_{2}\right)-\mathfrak{L}_{\mathcal{P}}^{-1}(i d-Q) \mathcal{N}_{\varepsilon} u\left(t_{1}\right) \mid \\
\leqslant & \frac{1}{\Gamma(\alpha)} \int_{0}^{t_{1}}\left[\psi^{\prime}(s)\left|\left(\psi\left(t_{2}\right)-\psi(s)\right)^{\alpha-1}-\left(\psi\left(t_{1}\right)-\psi(s)\right)^{\alpha-1}\right||h(s, u(s), u(\varepsilon s))|\right] \mathrm{d} s \\
& +\frac{1}{\Gamma(\alpha)} \int_{t_{1}}^{t_{2}} \psi^{\prime}(s)\left(\psi\left(t_{2}\right)-\psi(s)\right)^{\alpha-1}|h(s, u(s), u(\varepsilon s))| \mathrm{d} s \\
& +\frac{\left[\left(\psi\left(t_{2}\right)-\psi(0)\right)^{\alpha}-\left(\psi\left(t_{1}\right)-\psi(0)\right)^{\alpha}\right]}{\Gamma(\alpha)(\psi(\mathfrak{b})-\psi(0))^{\alpha}} \\
& \times \int_{0}^{\mathfrak{b}} \psi^{\prime}(s)(\psi(\mathfrak{b})-\psi(s))^{\alpha-1}|h(s, u(s), u(\varepsilon s))| \mathrm{d} s \\
\leqslant & \frac{1}{\Gamma(\alpha)} \int_{0}^{t_{1}} \psi^{\prime}(s)\left(\psi\left(t_{1}\right)-\psi(s)\right)^{\alpha-1}|h(s, u(s), u(\varepsilon s))-h(s, 0,0)| \mathrm{d} s \\
& -\frac{1}{\Gamma(\alpha)} \int_{0}^{t_{1}} \psi^{\prime}(s)\left(\psi\left(t_{2}\right)-\psi(s)\right)^{\alpha-1}|h(s, u(s), u(\varepsilon s))-h(s, 0,0)| \mathrm{d} s
\end{aligned}
$$




$$
\begin{aligned}
& +\frac{1}{\Gamma(\alpha)} \int_{0}^{t_{1}} \psi^{\prime}(s)\left[\left(\psi\left(t_{1}\right)-\psi(s)\right)^{\alpha-1}-\left(\psi\left(t_{2}\right)-\psi(s)\right)^{\alpha-1}\right]|h(s, 0,0)| \mathrm{d} s \\
& +\frac{1}{\Gamma(\alpha)} \int_{t_{1}}^{t_{2}} \psi^{\prime}(s)\left(\psi\left(t_{2}\right)-\psi(s)\right)^{\alpha-1}|h(s, u(s), u(\varepsilon s))-h(s, 0,0)| \mathrm{d} s \\
& +\frac{1}{\Gamma(\alpha)} \int_{t_{1}}^{t_{2}} \psi^{\prime}(s)\left(\psi\left(t_{2}\right)-\psi(s)\right)^{\alpha-1}|h(s, 0,0)| \mathrm{d} s \\
& +\frac{\left[\left(\psi\left(t_{2}\right)-\psi(0)\right)^{\alpha}-\left(\psi\left(t_{1}\right)-\psi(0)\right)^{\alpha}\right]}{\Gamma(\alpha)(\psi(\mathfrak{b})-\psi(0))^{\alpha}} \\
& \times \int_{0}^{\mathfrak{b}} \psi^{\prime}(s)(\psi(\mathfrak{b})-\psi(s))^{\alpha-1}|h(s, u(s), u(\varepsilon s))-h(s, 0,0)| \mathrm{d} s \\
& +\frac{\left[\left(\psi\left(t_{2}\right)-\psi(0)\right)^{\alpha}-\left(\psi\left(t_{1}\right)-\psi(0)\right)^{\alpha}\right]}{\Gamma(\alpha)(\psi(\mathfrak{b})-\psi(0))^{\alpha}} \int_{0}^{\mathfrak{b}} \psi^{\prime}(s)(\psi(\mathfrak{b})-\psi(s))^{\alpha-1}|h(s, 0,0)| \mathrm{d} s \\
& \leqslant 2 \Lambda\left(\psi\left(t_{2}\right)-\psi\left(t_{1}\right)\right)^{\alpha}+\Lambda\left[\left(\psi\left(t_{1}\right)-\psi(0)\right)^{\alpha}-\left(\psi\left(t_{2}\right)-\psi(0)\right)^{\alpha}\right] \\
& +\Lambda\left[\left(\psi\left(t_{2}\right)-\psi(0)\right)^{\alpha}-\left(\psi\left(t_{1}\right)-\psi(0)\right)^{\alpha}\right] \\
& \leqslant 2 \Lambda\left(\psi\left(t_{2}\right)-\psi\left(t_{1}\right)\right)^{\alpha}
\end{aligned}
$$

where

$$
\Lambda=\frac{h^{*}+(\gamma+\eta) \mathcal{M}}{\Gamma(\alpha+1)}
$$

Since the right-hand side of the above inequality tends to zero as $t_{1} \rightarrow t_{2}$ and the limit is independent of $u$, the operator $\mathfrak{L}_{\mathcal{P}}^{-1}(i d-Q) \mathcal{N}_{\varepsilon}(\bar{\Omega})$ is equicontinuous. By the Arzelà-Ascoli theorem, $\mathfrak{L}_{\mathcal{P}}^{-1}(i d-Q) \mathcal{N}_{\varepsilon}(\bar{\Omega})$ is relatively compact in $\mathcal{X}$. As a consequence of Steps $1-3$, we see that $\mathcal{N}_{\varepsilon}$ is $\mathfrak{L}$-compact in $\bar{\Omega}$, which completes the proof of the lemma.

Lemma 3.4 In addition to condition (H1), assume that

$$
\frac{(\gamma+\eta)}{\Gamma(\alpha+1)}(\psi(\mathfrak{b})-\psi(0))^{\alpha}<\frac{1}{2} .
$$

Then, there exists $\mathcal{A}>0$, which is independent of $\zeta$, such that

$$
\mathfrak{L}(u)-\mathcal{N}_{\varepsilon}(u)=-\zeta\left[\mathfrak{L}(u)+\mathcal{N}_{\varepsilon}(-u)\right] \text { implies }\|u\|_{\mathcal{X}} \leqslant \mathcal{A}, \zeta \in(0,1] .
$$

Proof Let $u \in \mathcal{X}$ satisfy

$$
\mathfrak{L}(u)-\mathcal{N}_{\varepsilon}(u)=-\zeta \mathfrak{L}(u)-\zeta \mathcal{N}_{\varepsilon}(-u)
$$

then

$$
\mathfrak{L}(u)=\frac{1}{1+\zeta} \mathcal{N}_{\varepsilon}(u)-\frac{\zeta}{1+\zeta} \mathcal{N}_{\varepsilon}(-u)
$$

From the expressions for $\mathfrak{L}$ and $\mathcal{N}_{\varepsilon}$, for any $t \in J$

$$
\mathfrak{L} u(t)={ }^{c} \mathfrak{D}_{0^{+}}^{\alpha ; \psi} u(t)=\frac{1}{1+\zeta} h(t, u(t), u(\varepsilon t))-\frac{\zeta}{1+\zeta} h(t,-u(t),-u(\varepsilon t)) .
$$

By applying $\mathfrak{I}_{0^{+}}^{\alpha ; \psi}$ to both sides of the above equation, we have

$$
\mathfrak{I}_{0^{+}}^{\alpha ; \psi} \mathfrak{D}_{0^{+}}^{\alpha ; \psi} u(t)=\mathfrak{I}_{0^{+}}^{\alpha ; \psi}\left(\frac{1}{1+\zeta} h(s, u(s), u(\varepsilon s))-\frac{\zeta}{1+\zeta} h(s,-u(s),-u(\varepsilon s))\right)(t) .
$$


Since the operator $\mathfrak{I}_{0^{+}}^{\alpha ; \psi}$ is linear

$$
\mathfrak{I}_{0^{+}}^{\alpha ; \psi} c \mathfrak{D}_{0^{+}}^{\alpha ; \psi} u(t)=\frac{1}{\zeta+1}\left[\mathfrak{I}_{0^{+}}^{\alpha ; \psi}(h(s, u(s), u(\varepsilon s)))(t)-\zeta \mathfrak{I}_{0^{+}}^{\alpha ; \psi}(h(s,-u(s),-u(\varepsilon s)))(t)\right] .
$$

Now, using Lemma 2.5 gives

$$
u(t)=u(0)+\frac{1}{\zeta+1}\left[\mathfrak{I}_{0^{+}}^{\alpha ; \psi}(h(s, u(s), u(\varepsilon s)))(t)-\zeta \mathfrak{I}_{0^{+}}^{\alpha ; \psi}(h(s,-u(s),-u(\varepsilon s)))(t)\right] .
$$

Thus, for every $t \in J$

$$
\begin{aligned}
|u(t)| \leqslant & |u(0)|+\frac{1}{(\zeta+1) \Gamma(\alpha)} \int_{0}^{t} \psi^{\prime}(s)(\psi(t)-\psi(s))^{\alpha-1}|h(s, u(s), u(\varepsilon s))| \mathrm{d} s \\
& +\frac{\zeta}{(\zeta+1) \Gamma(\alpha)} \int_{0}^{t} \psi^{\prime}(s)(\psi(t)-\psi(s))^{\alpha-1}|h(s,-u(s),-u(\varepsilon s))| \mathrm{d} s \\
\leqslant & |u(0)| \\
& +\frac{1}{(\zeta+1) \Gamma(\alpha)} \int_{0}^{t} \psi^{\prime}(s)(\psi(t)-\psi(s))^{\alpha-1}|h(s, u(s), u(\varepsilon s))-h(s, 0,0)| \mathrm{d} s \\
& +\frac{1}{(\zeta+1) \Gamma(\alpha)} \int_{0}^{t} \psi^{\prime}(s)(\psi(t)-\psi(s))^{\alpha-1}|h(s, 0,0)| \mathrm{d} s \\
& +\frac{\zeta}{(\zeta+1) \Gamma(\alpha)} \int_{0}^{t} \psi^{\prime}(s)(\psi(t)-\psi(s))^{\alpha-1}|h(s,-u(s),-u(\varepsilon s))-h(s, 0,0)| \mathrm{d} s \\
& +\frac{\zeta}{(\zeta+1) \Gamma(\alpha)} \int_{0}^{t} \psi^{\prime}(s)(\psi(t)-\psi(s))^{\alpha-1}|h(s, 0,0)| \mathrm{d} s \\
\leqslant & |u(0)|+\frac{2 h^{*}(\psi(\mathfrak{b})-\psi(0))^{\alpha}}{\Gamma(\alpha+1)}+\frac{2(\gamma+\eta)}{\Gamma(\alpha+1)}(\psi(\mathfrak{b})-\psi(0))^{\alpha}\|u\| \mathcal{X},
\end{aligned}
$$

where $h^{*}=\|h(\cdot, 0,0)\|_{\infty}$ as in Step 2 of the proof of Lemma 3.3. Therefore

$$
\|u\|_{\mathcal{X}} \leqslant|u(0)|+\frac{2 h^{*}(\psi(\mathfrak{b})-\psi(0))^{\alpha}}{\Gamma(\alpha+1)}+\frac{2(\gamma+\eta)}{\Gamma(\alpha+1)}(\psi(\mathfrak{b})-\psi(0))^{\alpha}\|u\|_{\mathcal{X}},
$$

and so

$$
\|u\|_{\mathcal{X}} \leqslant \frac{|u(0)|+\frac{2 h^{*}(\psi(\mathfrak{b})-\psi(0))^{\alpha}}{\Gamma(\alpha+1)}}{\left[1-\frac{2(\gamma+\eta)}{\Gamma(\alpha+1)}(\psi(\mathfrak{b})-\psi(0))^{\alpha}\right]}:=\mathcal{A} .
$$

This proves the lemma.

Lemma 3.5 If conditions (H1) and (6) hold, then there exist a bounded open set $\Omega \subset \mathcal{X}$ with

$$
\mathfrak{L}(u)-\mathcal{N}_{\varepsilon}(u) \neq-\zeta\left[\mathfrak{L}(u)+\mathcal{N}_{\varepsilon}(-u)\right],
$$

for any $u \in \partial \Omega$ and any $\zeta \in(0,1]$.

Proof Using Lemma 3.4, there exists a positive constant $\mathcal{A}$ independent of $\zeta$, such that, if $u$ satisfies

$$
\mathfrak{L}(u)-\mathcal{N}_{\varepsilon}(u)=-\zeta\left[\mathfrak{L}(u)+\mathcal{N}_{\varepsilon}(-u)\right], \zeta \in(0,1],
$$

then $\|u\|_{\mathcal{X}} \leqslant \mathcal{A}$. Thus, if

$$
\Omega=\left\{u \in \mathcal{X}:\|u\|_{\mathcal{X}}<\vartheta\right\}
$$

with $\vartheta>\mathcal{A}$, then

$$
\mathfrak{L}(u)-\mathcal{N}_{\varepsilon}(u) \neq-\zeta\left[\mathfrak{L}(u)-\mathcal{N}_{\varepsilon}(-u)\right],
$$

for all $u \in \partial \Omega=\left\{u \in \mathcal{X} ;\|u\|_{\mathcal{X}}=\vartheta\right\}$ and $\zeta \in(0,1]$, which is what we wanted to show.

Our first existence result is given in the following theorem. 
Theorem 3.6 If (H1) and (6) hold, then there exist at least one solution to the problem (1)-(2) in Dom $\mathfrak{L} \cap \bar{\Omega}$.

Proof It is clear that the set $\Omega$ defined in (8) is symmetric, $0 \in \Omega$, and $\mathcal{X} \cap \bar{\Omega}=\bar{\Omega} \neq \emptyset$. By Lemma 3.5

$$
\mathfrak{L}(u)-\mathcal{N}_{\varepsilon}(u) \neq-\zeta\left[\mathfrak{L}(u)-\mathcal{N}_{\varepsilon}(-u)\right]
$$

for each $u \in \mathcal{X} \cap \partial \Omega=\partial \Omega$ and each $\zeta \in(0,1]$. By Lemma 2.11, problem (1)-(2) has at least one solution in Dom $\mathfrak{L} \cap \bar{\Omega}$.

Next, we have a uniqueness result.

Theorem 3.7 In addition to (H1), assume that

(H2) There exist constants $\bar{\gamma}>0$ and $\bar{\eta} \geqslant 0$, such that

$$
|h(t, u, v)-h(t, \bar{u}, \bar{v})| \geqslant \bar{\gamma}|u-\bar{u}|-\bar{\eta}|v-\bar{v}|,
$$

for every $t \in J$ and $u, \bar{u}, v, \bar{v} \in \mathbb{R}$.

If

$$
\frac{\bar{\eta}}{\bar{\gamma}}+\frac{2(\gamma+\eta)}{\Gamma(\alpha+1)}(\psi(\mathfrak{b})-\psi(0))^{\alpha}<1
$$

then the problem (1)-(2) has a unique solution in Dom $\mathfrak{L} \cap \bar{\Omega}$.

Proof Since condition (6) must hold, by Theorem 3.6, the problem (1)-(2) has at least one solution in Dom $\mathfrak{L} \cap$ $\bar{\Omega}$.

To prove uniqueness of the solution, suppose that problem (1)-(2) has two different solutions $u_{1}, u_{2} \in$ $\operatorname{Dom} \mathfrak{L} \cap \bar{\Omega}$. Then, for each $t \in J$

$$
\begin{aligned}
{ }^{c} \mathfrak{D}_{0^{+}}^{\alpha ; \psi} u_{1}(t) & =h\left(t, u_{1}(t), u_{1}(\varepsilon t)\right), \\
{ }^{c} \mathfrak{D}_{0^{+}}^{\alpha ; \psi} u_{2}(t) & =h\left(t, u_{2}(t), u_{2}(\varepsilon t)\right), \\
u_{1}(0) & =u_{1}(\mathfrak{b}), \text { and } u_{2}(0)=u_{2}(\mathfrak{b}) .
\end{aligned}
$$

Let $u(t)=u_{1}(t)-u_{2}(t)$, for all $t \in J$. Then

$$
\begin{aligned}
\mathfrak{L} u(t) & ={ }^{c} \mathfrak{D}_{0^{+}}^{\alpha ; \psi} u(t) \\
& ={ }^{c} \mathfrak{D}_{0^{+}}^{\alpha ; \psi} u_{1}(t)-{ }^{c} \mathfrak{D}_{0^{+}}^{\alpha ; \psi} u_{2}(t) \\
& =h\left(t, u_{1}(t), u_{1}(\varepsilon t)\right)-h\left(t, u_{2}(t), u_{2}(\varepsilon t)\right) .
\end{aligned}
$$

Using the fact that $\operatorname{Img} \mathfrak{L}=\operatorname{ker} Q$, we have

$$
\int_{0}^{\mathfrak{b}} \psi^{\prime}(s)(\psi(\mathfrak{b})-\psi(s))^{\alpha-1}\left[h\left(s, u_{1}(s), u_{1}(\varepsilon s)\right)-h\left(s, u_{2}(s), u_{2}(\varepsilon s)\right)\right] \mathrm{d} s=0 .
$$

Since $h$ is continuous, there exists $t_{0} \in[0, \mathfrak{b}]$, such that

$$
h\left(t_{0}, u_{1}\left(t_{0}\right), u_{1}\left(\varepsilon t_{0}\right)\right)-h\left(t_{0}, u_{2}\left(t_{0}\right), u_{2}\left(\varepsilon t_{0}\right)\right)=0 .
$$

In view of (H2), we have

$$
\left|u_{1}\left(t_{0}\right)-u_{2}\left(t_{0}\right)\right| \leqslant \frac{\bar{\eta}}{\bar{\gamma}}\left|u_{1}\left(\varepsilon t_{0}\right)-u_{2}\left(\varepsilon t_{0}\right)\right| \leqslant \frac{\bar{\eta}}{\bar{\gamma}}\left\|u_{1}-u_{2}\right\|_{\mathcal{X}},
$$

so

$$
\left|u\left(t_{0}\right)\right| \leqslant \frac{\bar{\eta}}{\gamma}\|u\|_{\mathcal{X}} .
$$

On the other hand, by Lemma 2.5, we have

$$
\mathfrak{I}_{0^{+}}^{\alpha ; \psi} \mathfrak{D}_{0^{+}}^{\alpha ; \psi} u(t)=u(t)-u(0),
$$


which implies that

$$
u(0)=u\left(t_{0}\right)-\mathfrak{I}_{0^{+}}^{\alpha ; \psi} c \mathfrak{D}_{0^{+}}^{\alpha ; \psi} u\left(t_{0}\right)
$$

and therefore

$$
u(t)=\mathfrak{I}_{0^{+}}^{\alpha ; \psi} \mathfrak{D}_{0^{+}}^{\alpha ; \psi} u(t)+u\left(t_{0}\right)-\mathfrak{I}_{0^{+}}^{\alpha ; \psi} \mathfrak{D}_{0^{+}}^{\alpha ; \psi} u\left(t_{0}\right) .
$$

Using (11), for every $t \in J$

$$
\begin{aligned}
|u(t)| & \leqslant\left|\mathfrak{I}_{0^{+}}^{\alpha ; \psi}{ }^{c} \mathfrak{D}_{0^{+}}^{\alpha ; \psi} u(t)\right|+\left|u\left(t_{0}\right)\right|+\left|\mathfrak{I}_{0^{+}}^{\alpha ; \psi}{ }^{+} \mathfrak{D}_{0^{+}}^{\alpha ; \psi} u\left(t_{0}\right)\right| \\
& \leqslant \frac{\bar{\eta}}{\bar{\gamma}}\|u\|_{\mathcal{X}}+\frac{2(\psi(\mathfrak{b})-\psi(0))^{\alpha}}{\Gamma(\alpha+1)}\left\|{ }^{c} \mathfrak{D}_{0^{+}}^{\alpha ; \psi} u\right\|_{\mathcal{X}} .
\end{aligned}
$$

From (10) and (H1), we see that

$$
\left|{ }^{c} \mathfrak{D}_{0^{+}}^{\alpha ; \psi} u(t)\right|=\left|h\left(t, u_{1}(t), u_{1}(\varepsilon t)\right)-h\left(t, u_{2}(t), u_{2}(\varepsilon t)\right)\right| \leqslant(\gamma+\eta)\|u\|_{\mathcal{X}}
$$

and so

$$
\left\|c \mathfrak{D}_{0^{+}}^{\alpha ; \psi} u\right\|_{\mathcal{X}} \leqslant(\gamma+\eta)\|u\|_{\mathcal{X}} .
$$

Substituting (13) into the right-hand side of (12), we obtain

$$
|u(t)| \leqslant\left[\frac{\bar{\eta}}{\bar{\gamma}}+\frac{2(\gamma+\eta)(\psi(\mathfrak{b})-\psi(0))^{\alpha}}{\Gamma(\alpha+1)}\right]\|u\|_{\mathcal{X}}
$$

for every $t \in J$. Therefore

$$
\|u\|_{\mathcal{X}} \leqslant\left[\frac{\bar{\eta}}{\bar{\gamma}}+\frac{2(\gamma+\eta)(\psi(\mathfrak{b})-\psi(0))^{\alpha}}{\Gamma(\alpha+1)}\right]\|u\|_{\mathcal{X}}
$$

which by (9) implies

$$
\|u\|_{\mathcal{X}}=0 .
$$

That is, for any $t \in J, u(t)=0$ or $u_{1}(t)=u_{2}(t)$. This completes the proof.

\section{Examples}

Example 1 Consider the problem

$$
\left\{\begin{array}{l}
c \mathfrak{D}_{0^{+}}^{\frac{1}{3} ; 2^{t}} u(t)=h(t, u(t), u(\varepsilon t)), \quad t \in J:=[0,1], \\
u(0)=u(1),
\end{array}\right.
$$

where

$$
h(t, u(t), u(\varepsilon t))=\frac{u(t)}{5(1+t)}+\frac{1}{3 \sqrt{\pi}} \sin u\left(\frac{t}{\sqrt{2}}\right)+\frac{e^{t}}{3} .
$$

Here $\alpha=\frac{1}{3}, \psi(t)=2^{t}$, and $\varepsilon=\frac{1}{\sqrt{2}}$. Clearly, $h \in C([0,1] \times \mathbb{R} \times \mathbb{R}, \mathbb{R})$. Let $u, \bar{u}, v, \bar{v} \in \mathbb{R}$, and $t \in J$; then

$$
|h(t, u, v)-h(t, \bar{u}, \bar{v})| \leqslant \frac{1}{5}|u-\bar{u}|+\frac{1}{3 \sqrt{\pi}}|v-\bar{v}|,
$$

so (H1) is satisfied with $\gamma=\frac{1}{5}$ and $\eta=\frac{1}{3 \sqrt{\pi}}$. By a simple calculation, we see that

$$
\frac{(\gamma+\eta)}{\Gamma(\alpha+1)}(\psi(\mathfrak{b})-\psi(0))^{\alpha} \approx 0.4346<\frac{1}{2} .
$$

Therefore, by Theorem 3.6, this problem has at least one solution. 
Example 2 Consider the problem

$$
\left\{\begin{array}{l}
c \mathfrak{D}_{0^{+}}^{\frac{1}{2} ; e^{t}} u(t)=h(t, u(t), u(\varepsilon t)), \quad t \in J:=[0,1] \\
u(0)=u(1)
\end{array}\right.
$$

where

$$
h(t, u(t), u(\varepsilon t))=\ln (t+2)+\frac{1}{23 \sqrt{\pi}}\left(\sin u(t)+\frac{3}{2} u(t)\right)+\frac{e^{-11-t}}{37\left(1+u\left(\frac{t}{\sqrt{\pi}}\right)\right)} .
$$

Here, $\alpha=\frac{1}{2}, \psi(t)=e^{t}$, and $\varepsilon=\frac{1}{\sqrt{\pi}}$. It is easy to see that $h \in C([0,1] \times \mathbb{R} \times \mathbb{R}, \mathbb{R})$. For $u, \bar{u}, v, \bar{v} \in \mathbb{R}$ and $t \in J$

$$
|h(t, u, v)-h(t, \bar{u}, \bar{v})| \leqslant \frac{5}{46 \sqrt{\pi}}|u-\bar{u}|+\frac{1}{37 e^{11}}|v-\bar{v}|
$$

and

$$
|h(t, u, v)-h(t, \bar{u}, \bar{v})| \geqslant \frac{1}{46 \sqrt{\pi}}|u-\bar{u}|-\frac{1}{37 e^{11}}|v-\bar{v}| .
$$

Hence, (H1) and (H2) are satisfied with

$$
\gamma=\frac{5}{46 \sqrt{\pi}}, \bar{\gamma}=\frac{1}{46 \sqrt{\pi}}, \eta=\bar{\eta}=\frac{1}{37 e^{11}} .
$$

A calculation shows that

$$
\frac{\bar{\eta}}{\bar{\gamma}}+\frac{2(\gamma+\eta)}{\Gamma(\alpha+1)}(\psi(\mathfrak{b})-\psi(0))^{\alpha} \approx 0.1814<1,
$$

so by Theorem 3.7, the problem has a unique solution.

\section{Conclusions}

Using Mawhin's [14] coincidence degree theory, we obtained the existence and uniqueness of solutions to the nonlinear fractional pantograph differential equations involving the $\psi$-Caputo derivative. The results are illustrated with examples. It is worth noting that not only do we present a new class of fractional differential equations involving the $\psi$-Caputo fractional derivative, but by choosing $\psi(t)=t$ or $\psi(t)=\ln t$, we have existence results for problems involving the Caputo or the Caputo-Hadamard type fractional derivatives, respectively.

Acknowledgements The authors are grateful to the referees for their helpful remarks and suggestions.

Open Access This article is licensed under a Creative Commons Attribution 4.0 International License, which permits use, sharing, adaptation, distribution and reproduction in any medium or format, as long as you give appropriate credit to the original author(s) and the source, provide a link to the Creative Commons licence, and indicate if changes were made. The images or other third party material in this article are included in the article's Creative Commons licence, unless indicated otherwise in a credit line to the material. If material is not included in the article's Creative Commons licence and your intended use is not permitted by statutory regulation or exceeds the permitted use, you will need to obtain permission directly from the copyright holder. To view a copy of this licence, visit http://creativecommons.org/licenses/by/4.0/.

\section{References}

1. Abbas, S.; Benchohra, M.; N'Guérékata, G.M.: Topics in Fractional Differential Equations. Springer, New York (2012)

2. Abbas, S.; Benchohra, M.; N'Guérékata, G.M.: Advanced Fractional Differential and Integral Equations. Nova Science Publishers, New York (2014)

3. Abdo, M.S.; Abdeljawad, T.; Kucche, K.D.; Alqudah, M.A.; Ali, S.M.; Jeelani, M.B.: On nonlinear pantograph fractional differential equations with Atangana-Baleanu-Caputo derivative. Adv. Differ. Equ. 2021(65), 1-17 (2021)

4. Agrawal, O.P.: Some generalized fractional calculus operators and their applications in integral equations. Frac. Cal. Appl. Anal. 15, 700-711 (2012) 
5. Almeida, R.: A Caputo fractional derivative of a function with respect to another function. Commun. Nonlinear Sci. Numer. Simul. 44, 460-481 (2017)

6. Almeida, R.: Functional differential equations involving the $\psi$-Caputo fractional derivative. Fract. Fract. 4(29), 1-8 (2020)

7. Almeida, R.; Malinowska, A.B.; Odzijewicz, T.: On systems of fractional differential equations with the $\psi$-Caputo derivative and their applications. Math. Meth. Appl. Sci. 44, 1-16 (2019)

8. Balachandran, K.; Kiruthika, S.; Trujillo, J.J.: Existence of solutions of nonlinear fractional pantograph equations. Acta. Math. Sci. Sci. Ser. B (Engl. Ed.) 33, 712-720 (2013)

9. Benchohra, M.; Bouriah, S.; Henderson, J.: Existence and stability results for nonlinear implicit neutral fractional differential equations with finite delay and impulses. Commun. Appl. Nonlinear Anal. 22, 46-67 (2015)

10. Benchohra, M.; Bouriah, S.: Existence and stability results for nonlinear boundary value problem for implicit differential equations of fractional order, Moroccan J. Pure. Appl. Anal. 1, 22-36 (2015)

11. Benchohra, M.; Bouriah, S.; Nieto, J.J.: Existence of periodic solutions for nonlinear implicit Hadamard's fractional differential equations. Rev. R. Acad. Cienc. Exactas Fs. Nat. Ser. A Mat. RACSAM 112, 25-35 (2018)

12. Benchohra, M.; Bouriah, S.; Graef, J.R.: Nonlinear implicit differential equation of fractional order at resonance. Electron. J. Differ. Equ. 2016(324), 1-10 (2016)

13. Derbazi, C.; Baitiche, Z.: Coupled systems of $\psi$-Caputo differential equations with initial conditions in Banach spaces. Mediter. J. Math. 17(169), 13 (2020)

14. Gaines, R.E.; Mawhin, J.: Coincidence Degree and Nonlinear Differential Equations, vol. 568. Lecture Notes in Math. Springer, Berlin (1977)

15. Herrmann, R.: Fractional Calculus: An Introduction for Physicists. World Scientific, Singapore (2011)

16. Hilfer, R.: Applications of Fractional Calculus in Physics. World Scientific, Singapore (2000)

17. Kilbas, A.A.; Srivastava, H.M.; Trujillo, J.J.: Theory and Applications of Fractional Differential Equations, North-Holland Mathematics Studies 204. Elsevier Science B.V, Amsterdam (2006)

18. Mawhin, J.: Topological Degree Methods in Nonlinear Boundary Value Problems, CBMS Regional Conference Series in Mathematics, 40. American Mathematical Society, Providence, R.I. (1979)

19. O’Regan, D.; Chao, Y.J.; Chen, Y.Q.: Topological Degree Theory and Application. Taylor and Francis, Boca Raton (2006)

20. Rahimkhani, P.; Ordokhani, Y.; Babolian, E.: Numerical solution of fractional pantograph differential equations by using generalized fractional-order Bernoulli wavelet. J. Comput. Appl. Math. 309, 493-510 (2017)

21. Saeed, U.; Rehman, M.: Hermite wavelet method for fractional delay differential equations. J. Differ. Equ. 2014, 8 (2014) Article ID 359093

22. Samko, S.G.; Kilbas, A.A.; Marichev, O.I.: Fractional Integrals and Derivatives. Theory and Applications. Gordon and Breach, Yverdon (1993)

23. Shah, K.; Vivek, D.; Kanagarajan, K.: Dynamics and stability of $\psi$-fractional pantograph equations with boundary conditions. Bol. Soc. Paran. Mat. 39, 43-55 (2021)

Publisher's Note Springer Nature remains neutral with regard to jurisdictional claims in published maps and institutional affiliations. 